\title{
Shedding of Staphylococcus aureus and methicillin-resistant Staphylococcus aureus from adult and pediatric bathers in marine waters
}

\author{
Lisa RW Plano 1,2*, Anna C Garza', Tomoyuki Shibata ${ }^{2,3,4}$, Samir M Elmir ${ }^{2,5}$, Jonathan Kish ${ }^{2,6}$, \\ Christopher D Sinigalliano ${ }^{2,3}$, Maribeth L Gidley ${ }^{2,3}$, Gary Miller ${ }^{5}$, Kelly Withum², Lora E Fleming ${ }^{2,6}$, \\ Helena M Solo-Gabriele ${ }^{2,7}$
}

\begin{abstract}
Background: Staphylococcus aureus including methicillin resistant S. aureus, MRSA, are human colonizing bacteria that commonly cause opportunistic infections primarily involving the skin in otherwise healthy individuals. These infections have been linked to close contact and sharing of common facilities such as locker rooms, schools and prisons Waterborne exposure and transmission routes have not been traditionally associated with S. aureus infections. Coastal marine waters and beaches used for recreation are potential locations for the combination of high numbers of people with close contact and therefore could contribute to the exposure to and infection by these organisms. The primary aim of this study was to evaluate the amount and characteristics of the shedding of methicillin sensitive S. aureus, MSSA and MRSA by human bathers in marine waters.
\end{abstract}

Results: Nasal cultures were collected from bathers, and water samples were collected from two sets of pools designed to isolate and quantify MSSA and MRSA shed by adults and toddlers during exposure to marine water. A combination of selective growth media and biochemical and polymerase chain reaction analysis was used to identify and perform limited characterization of the $S$. aureus isolated from the water and the participants. Twelve of 15 MRSA isolates collected from the water had identical genetic characteristics as the organisms isolated from the participants exposed to that water while the remaining 3 MRSA were without matching nasal isolates from participants. The amount of $S$. aureus shed per person corresponded to $10^{5}$ to $10^{6} \mathrm{CFU}$ per person per 15 -minute bathing period, with 15 to $20 \%$ of this quantity testing positive for MRSA.

Conclusions: This is the first report of a comparison of human colonizing organisms with bacteria from human exposed marine water attempting to confirm that participants shed their own colonizing MSSA and MRSA into their bathing milieu. These findings clearly demonstrate that adults and toddlers shed their colonizing organisms into marine waters and therefore can be sources of potentially pathogenic S. aureus and MRSA in recreational marine waters. Additional research is needed to evaluate recreational beaches and marine waters as potential exposure and transmission pathways for MRSA.

\section{Background}

Staphyloccus aureus is an opportunistic pathogen capable of causing a wide variety of infectious diseases and is usually associated with humans as commensal colonizing organisms in at least $30 \%$ of the population [1-3]. Staphylococcal infections are primarily of the skin and

\footnotetext{
* Correspondence: Iplano@med.miami.edu

'Department of Pediatrics and Department of Microbiology and

Immunology, University of Miami, Miami, Florida 33130 USA Full list of author information is available at the end of the article
}

soft tissues; however, they are capable of causing much more serious systemic infections and death, especially when associated with methicillin resistance $[4,5]$. Initially, outbreaks of methicillin resistant S. aureus (MRSA) infections were associated with hospitals and healthcareassociated exposures in compromised patients; however, since the late $1990 \mathrm{~s}$ with the emergence of new more aggressive community-associated MRSA (CA-MRSA), these infections are no longer limited to these settings.
C Biomed Central 
Since its emergence, outbreaks of CA-MRSA infections in otherwise young healthy individuals [6] have been linked to close contact and sharing of common facilities such as locker rooms, schools and prisons [7]. These community-associated strains have made their way into the hospitals [5], being responsible for a significant number of hospital-acquired infections; and in a recent report, they have now been shown to be responsible for the majority of skin infections requiring treatment in emergency departments in multiple U.S. cities [8].

Waterborne transmission routes have not been traditionally associated with $S$. aureus infections. However, in some earlier studies, investigators in Hawaii reported cases of $S$. aureus infections associated with exposure to coastal marine waters $[9,10]$, with humans serving as the suspected primary source [11]. They also showed that these organisms are able to remain viable in seawater over several days [12]. Therefore, coastal marine waters used for recreation could provide a transmission pathway for both colonization and/or infection of individuals. Previous studies have also identified $S$. aureus in recreational marine water [12,13], and S. aureus and MRSA in sand [14-16].

In an earlier study attempting to quantify $S$. aureus release by humans in marine water [17], investigators showed that humans shed greater quantities of $S$. aureus than the fecal indicator bacteria enterococci. However, this earlier study was limited in its methodology and criteria used to isolate and confirm $S$. aureus, and it did not address the potential presence of MRSA in the isolates. Furthermore, the study was also limited to an adult population, and it did not evaluate for $S$. aureus colonization of the human population studied.

As recreational marine waters and beaches may be commonly used by many people over the course of a short period of time, the risk of exposure to all microorganisms that are in this environment increases. Given that transmission of $S$. aureus (including MRSA) has been documented in settings associated with shared facilities and close contact, the use of recreational marine waters and beaches could certainly represent another possible route of exposure and transmission of these potentially pathogenic organisms and warrant investigation.

The aim of this study was to evaluate the amounts, as well as the characteristics, of $S$. aureus, methicillin sensitive $S$. aureus (MSSA), and MRSA shed by humans into recreational waters and sands. In this study, S. aureus, MSSA, and MRSA shed from adults, and for the first time children, were identified using stringent selection and identification procedures.

\section{Methods}

The study was approved by the Florida Department of Health Internal Review Board (IRB 1491; DOH IRB
Number, H07164) and the University of Miami Internal Review Board (IRB 20070306). Consent forms were signed by each study participant (or parent/guardian), and participant identity was kept confidential. The field experimental design followed that of Elmir et al. [17,18], including the use of the same study site (a sub-tropical non-point source recreational marine beach).

\section{Pool field studies}

The "Large Pool" field study was used to determine the total amount of $S$. aureus and the distribution of $S$. aureus relative to MSSA and MRSA released from the bodies of adult bathers $[17,18]$. Briefly, after filling an inflatable pool with $1400 \mathrm{~L}$ of local off-shore marine water (where no humans were observed swimming at the time of collection), two groups of 10 adult participants were subjected to a series of four continuous 15-minute bathing cycles conducted on a single day in July 2008 beginning at 9:00 AM and 12:00 PM, respectively. In between bathing cycles, the pool was cleaned and refilled from the same source water. Participants had no sand exposure during the first two cycles, but were exposed to beach sand during the last two cycles. Samples of the source water, pool water before participant contact (in triplicate) and pool water after participant contact (in triplicate) were collected after each cycle. Source water, pool water and residual sand samples were analyzed as described below. The demographic characteristics of the 20 adult "Large Pool" participants (10 males and 10 females) included an age range from 19 to 51 years old, and body weights ranging from 50 to $100 \mathrm{~kg}[18]$.

The "Small Pool" field study was used to determine the total amounts of $S$. aureus and the distribution of $S$. aureus among MSSA and MRSA released from the bodies of a pediatric population, including an estimate of the contribution from the sand adhered to the pediatric participant [18]. Briefly, in the same area of the beach as the adult studies during two days in July and August of 2008, 14 individual toddlers wearing bathing suits over diapers spent 15 to 30 minutes on the beach sand (e.g. playing, sitting, lying, walking, etc). Following sand exposure, toddlers were placed in a 190-liter tub, while local off-shore marine water $(14 \mathrm{~L})$ was poured from sanitized watering cans gently over their heads and bodies. When necessary the toddlers were held upright in pool by an adult with either gloved hands or hands sanitized with alcohol. Sanitation of the pool and sample collections (in triplicate) were performed as described [18]. Source water, pool water and residual sand samples were analyzed as described below. The demographic characteristics of the 14 "Small Pool" toddlers (2 males and 12 females) included ages ranging from 5 to 47 months, and weights ranging from 6.8 to $16.3 \mathrm{~kg}$ [18]. 
Prior to study initiation, nasal cultures were obtained from the anterior nares from all participants using rayon swabs (BBL culture swab: Becton, Dickinson and Company) and $S$. aureus were cultured as described below.

\section{Bacterial isolation and identification}

$S$. aureus was isolated from the water samples using a standard membrane filtration (MF) method [19], followed by growth on selective media, Baird Parker agar (Becton, Dickinson and Company, Sparks, MD) with Egg Yolk (EY) Tellurite Enrichment (Becton, Dickinson and Company), BP, and CHROMagar, CHR (Becton, Dickinson and Company) (see Figure 1 for process flow). MSSA and MRSA isolated from BP plates were subjected to genetic tests and compared to organisms isolated from nasal cultures. MSSA isolated from CHR plates was used for direct comparison of colony counts obtained during previous studies. Facilities used for processing samples were located within minutes from the study site, allowing for the processing of samples within one hour after collection. Volumes of the source water used for filtration were $10 \mathrm{ml}$ and $100 \mathrm{ml}$; volumes of the pool water samples used for filtration prior to and after adult participant contact were $10 \mathrm{ml}$ and $50 \mathrm{ml}$ respectively; volumes of the water used for filtration after contact with the pediatric participants were $5 \mathrm{ml}$, $10 \mathrm{ml}$, and $50 \mathrm{ml}$. Multiple volumes were filtered in order to obtain quantifiable colony counts as the levels of bacteria in both the source water and the experimental pool water samples were unknown.

The analysis of $S$. aureus in sand was similar to that for water with the exception of two pre-processing

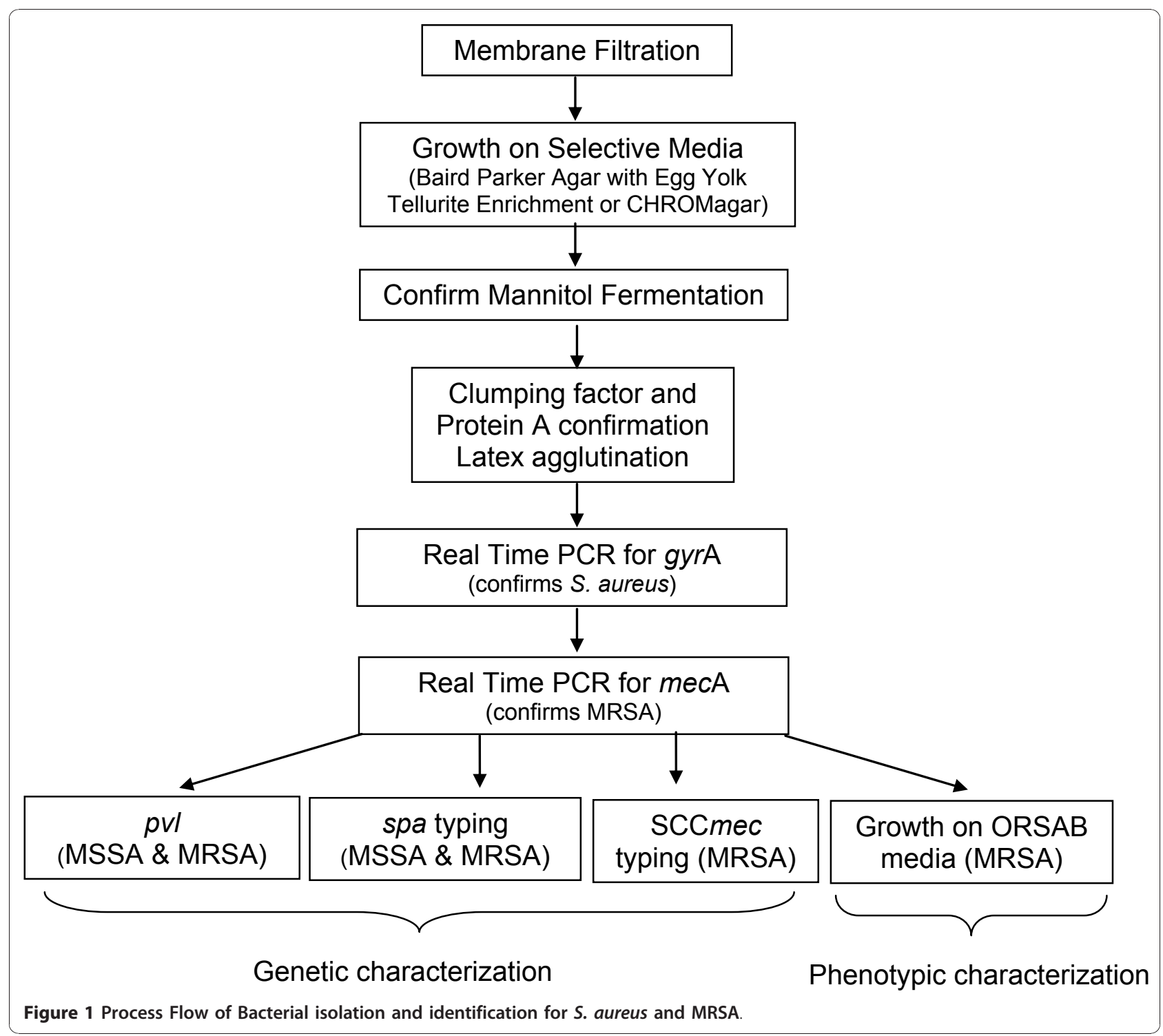


steps. The first step measured the water content of sand (weight difference of sand before and after drying at $110^{\circ} \mathrm{C}$ for $24 \mathrm{~h}$ ). The second step extracted bacteria from the sand particles to a predefined volume of sterile water. To accomplish this, pre-weighed un-dried sand was aseptically removed from the corresponding sample container and placed into a sterile pre-weighed jar. One hundred and ten milliliters of sterile phosphate buffer saline (PBS) were added to each jar, and the jars were shaken vigorously for 30 seconds. The samples were permitted to settle for 30 seconds, and the supernatant was subsequently used for membrane filtration. One hundred milliliters of the sand eluate samples were used for the filtration and bacterial quantification.

Following standard MF, filter membranes were placed on $\mathrm{BP}$ and $\mathrm{CHR}$, and incubated aerobically at $37^{\circ} \mathrm{C}$ for a minimum of $24 \mathrm{~h}$. After incubation, colonies found to be black, shiny, convex, 2-5 $\mathrm{mm}$ in diameter, and surrounded by clear zones (BP) or mauve (CHR), were considered presumptive $S$. aureus, and subjected to confirmatory tests. All presumptive positive isolates were transferred to Mannitol Salt agar (Becton, Dickinson and Company), for the determination of mannitol fermentation, and incubated aerobically at $37^{\circ} \mathrm{C}$ for $16-24 \mathrm{~h}$. All mannitol-fermenting isolates were enriched [20] on Trypticase Soy Agar with 5\% Sheep Blood (TSA II, Becton, Dickinson and Company) for determination of colony morphology and gross pigmentation, the ability to lyse red blood cells and to provide bacterial cells for latex agglutination tests for clumping factor and protein $\mathrm{A}$ using the Remel BactiStaph Latex Agglutination Test (Thermo Fisher Scientific, Lenexa, KS).

The analysis of the nasal swab cultures focused on detection and genetic characterization, rather than quantification. The method used was the same as that used for the water samples, except that the membrane filtration step was omitted. Utilizing standard aseptic techniques swabs were placed in $0.5 \mathrm{ml}$ tripticase soy broth TSB (Becton, Dickinson and Company) supplemented with $6.5 \%$ sodium chloride, and incubated overnight at $37^{\circ} \mathrm{C}$ for enrichment. One hundred micro-liters of the overnight broth were transferred to Mannitol Salt agar (Becton, Dickinson and Company), and the organisms were identified and confirmed as detailed above.

Chromosomal DNA was extracted from colonies isolated from water, sand, and nasal cultures. Whole cell extracts were prepared from latex agglutination positive bacterial isolates using the Amplicor MTB Sputum Specimen Preparation Kit (Roche Molecular Systems, Inc., Indianapolis, IN) according to the manufacture's recommendations, and used as template for confirming and characterizing polymerase chain reactions (PCR) as outlined below. These DNA extracts (up to a maximum of 22 per filter) were subjected to PCR analysis of the $S$. aureus specific gyrA gene for S. aureus confirmation and the mecA gene for genetic MRSA confirmation. Oligonucleotide primers and thermal cycling conditions were used as described previously [21], with the minor modification that 5- $\mu$ l of whole cell extract was used as template in initial PCR reactions instead of purified chromosomal DNA.

All organisms determined to be genotypic MRSA (testing positive for $m e c A$ ) were re-isolated from agar plates, and grown on oxacillin resistance screening agar base media ORSAB (Remel; Thermo Fisher Scientific), a selective media for confirmation of phenotypic MRSA. All genotypic MRSA isolates from this study showed the phenotypic characteristics of MRSA. All confirmed MRSA ( $\mathrm{n}=17)$ and MSSA $(\mathrm{n}=162)$ collected from water and sand samples and all nasal cultures were stored as stock strains at $-80^{\circ} \mathrm{C}$.

The number of colonies testing positive for gyrA gene (for S. aureus counts) and mecA gene (for MRSA counts) were reported. Counts were then adjusted to colony forming units per $100 \mathrm{ml}$ water (CFU/100 ml) or per $100 \mathrm{~g}$ sand (CFU/100 g) using the volume of water applied to the filters or the weight of the sand collected from the pool. The numbers of microbes shed per person were determined by multiplying the difference in microbial concentrations measured before and after bathing in the pools by the water volumes corresponding to each person.

\section{Genetic characterization}

Bacterial isolates determined to be positive for $S$. aureus specific gyrA and MRSA specific mecA were subjected to additional PCR to test for the toxin genes for PantonValentine leukocidin, $p v l$, to evaluate the pathogenic potential of isolated organisms as previously described [21]. Staphylococcus cassette chromosome methicillin, SCCmec, type was determined for all MRSA as described [22]; and Staphylococcus protein A, spa, type was determined for all MRSA and a representative subset of MSSA as described [23] and using RIDOM spa type server to analyze sequences. SCCmec and spa types were determined in order to provide specific genetic characteristics for comparison between the nasal isolates from the colonized participants and the water samples from the pools. Genetic characteristics were evaluated for all MRSA isolates and all MSSA isolates from the nasal swabs, and from the water and sand samples from the small pool. Due to the large number of $S$. aureus isolates from the large pool, genetic characterization was conducted on a representative set of the MSSA isolates from each large pool water collection and choosen to include a subset of all colony morphology, gross pigmentation and RBC hemolysis type present in each set. All MSSA collected from the small pool water samples from the single colonized pediatric participant were analyzed. 


\section{Statistical analyses}

Data analyses (including Pearson Correlations, Student T-Tests, and Sum Rank Tests) were performed using Microsoft Excel 2003 and Sigmaplot 11.

\section{Results}

\section{Off shore water quality}

The physical-chemical characteristics of the source water taken off shore were typical of marine waters in subtropical environments (salinity $=34 \mathrm{psu}, \mathrm{pH}=7.9$, temperature $=$ $31^{\circ} \mathrm{C}$ ). The concentrations of $S$. aureus in the source water samples prior to human exposure were primarily below the detection limit of 1 CFU/100 mL. Only 1 of 8 (13\%) samples measured at the detection limit of 1 CFU/100 mL using the MF method with selection on CHR. Two of 22 (9.1\%) samples measured at $10 \mathrm{CFU} / 100 \mathrm{~mL}$ using selection on BP. The concentrations of $S$. aureus in the pool before versus after bathing differed by two-orders-of-magnitude indicating that background levels of $S$. aureus in the source water was insignificant. MRSA was not detected from any source water samples. Overall, these results are consistent with earlier studies that showed that the offshore waters at the study site are characterized by low concentrations of viable indicator bacteria $[17,18,24]$.

\section{S. aureus released by bathers}

In the large pool study with adults, the total quantities of $S$. aureus released per person were lower $\left(10^{5}\right)$ by about an order of magnitude, in the first two bathing cycles as compared to Elmir et al. [17] who reported releases on the order of $10^{6}$ per person (Table 1). The results appeared to converge for the last two cycles at about $10^{5} \mathrm{CFU} /$ person released. On average for all four cycles and for both groups, $S$. aureus counts were $6.3 \times$ $10^{5} \mathrm{CFU} /$ person from BP selection which was $40 \%$ higher than $3.8 \times 10^{5} \mathrm{CFU} /$ person from $\mathrm{CHR}$ selection.

In the toddler studies carried out individually in the small pools, the total shedding of S. aureus was assumed to be the sum of the numbers observed in the sand component and in the water component. Based on the sand analysis using BP selection, the numbers of $S$. aureus transported per toddler via sand ranged from less than the detection limit (2 to $6 \mathrm{CFU} /$ person) to 500 $\mathrm{CFU} /$ person with an estimated average of $69+/-145$ CFU/person (Table 2). The estimated numbers of $S$. aureus (BP) shed per toddler based on the water analysis was higher, ranging from less than the detection limit (280 CFU/person) to $4.5 \times 10^{5} \mathrm{CFU} /$ person, with an average of $4.3 \times 10^{4}+/-1.2 \times 10^{5} \mathrm{CFU} /$ person. The high standard deviations of the sand and water results were due to a large number of samples measured at the detection limit of the method; however, when samples were positive, the detected levels were elevated. When evaluating the significance of the sand relative to the total amount shed, the sand contributions for the single "Small Pool" bathing cycle ranged from less than 0.1 to $1.8 \%$, with an estimated average of $0.32+/-0.0 .09 \%(\mathrm{n}=$ 10 subjects with sediment in the pool). Subjects were excluded from this comparison if $S$. aureus was not detected in both sediment and water samples.

\section{Distribution of MSSA and MRSA: Nasal Colonization and detection in water}

There were a total of 34 nasal cultures (20 from the adult participants and 14 from the toddler participants). A total of 4 adult study participants were determined to be colonized with $S$. aureus by nares cultures. Two participants in group I had nasal cultures that were positive for MSSA, and two in group II were positive for MRSA. Among the adult population evaluated, the majority of the $S$. aureus shed into the water was MSSA. No MRSA was detected from Group I adults. Two of the 10 adult bathers in Group II were colonized with MRSA, and the Group II pool water was the only water where MRSA was detected. Water from the three cycles from Group II tested positive for MRSA using BP selection, and water from the two cycles were positive for MRSA using CHR selection.

Table 1 Colony forming units of S. aureus shed per adult

\begin{tabular}{|c|c|c|c|c|c|c|c|}
\hline \multirow[b]{2}{*}{ Cycle } & \multicolumn{2}{|c|}{ Group I } & \multicolumn{2}{|c|}{ Group II } & \multicolumn{2}{|c|}{ Average } & \multirow[b]{2}{*}{ (CHR) } \\
\hline & (BP) & (CHR) & (BP) & (CHR) & (BP) & (CHR) & \\
\hline 1 & $1.3 \times 10^{6}$ & $8.1 \times 10^{5}$ & ${ }^{*} 1.4 \times 10^{5}$ & BDL & $7.1 \times 10^{5}$ & $4.1 \times 10^{5}$ & $6.1 \times 10^{6}$ \\
\hline 2 & $8.3 \times 10^{5}$ & $8.1 \times 10^{5}$ & $4.6 \times 10^{4}$ & BDL & $4.4 \times 10^{5}$ & $4.1 \times 10^{5}$ & $3.9 \times 10^{6}$ \\
\hline 3 & $9.1 \times 10^{5}$ & $4.2 \times 10^{5}$ & $* 1.0 \times 10^{6}$ & $* 4.3 \times 10^{5}$ & $9.6 \times 10^{5}$ & $4.3 \times 10^{5}$ & $1.3 \times 10^{6}$ \\
\hline 4 & $3.6 \times 10^{5}$ & $8.1 \times 10^{4}$ & $* 4.3 \times 10^{5}$ & $* 4.5 \times 10^{5}$ & $3.9 \times 10^{5}$ & $2.6 \times 10^{5}$ & $6.8 \times 10^{5}$ \\
\hline Average & $8.4 \times 10^{5}$ & $5.3 \times 10^{5}$ & $4.1 \times 10^{5}$ & $2.2 \times 10^{5}$ & $6.3 \times 10^{5}$ & $3.8 \times 10^{5}$ & $3.0 \times 10^{6}$ \\
\hline Standard Deviation & $3.8 \times 10^{5}$ & $3.5 \times 10^{5}$ & $4.4 \times 10^{5}$ & $2.5 \times 10^{5}$ & $2.7 \times 10^{5}$ & $7.6 \times 10^{4}$ & $2.5 \times 10^{6}$ \\
\hline
\end{tabular}

*Water samples where MRSA was detected.

Based on analysis of water samples from a large pool with 10 adults using two different selective media, Baird Parker (BP) and Chromagar (CHR); numbers shown correspond to confirmed numbers of $S$. aureus based upon detection of the specific gyrA gene. BDL = below detection limit. BDLs were transformed to $1 / 2$ of $\mathrm{BDLs}$ in order to estimate averages and standard deviations. The theoretical detection limit $\left(2.8 \times 10^{3} \mathrm{CFU} /\right.$ person) can be calculated from the volume of the large pool $(1400 \mathrm{~L})$, the largest membrane filtration volume $(50 \mathrm{ml})$ and noting that 10 people bathed per cycle (adapted Elmir et al. [17]). 
Table 2 Colony forming units of S. aureus shed per toddler

\begin{tabular}{|c|c|c|c|c|}
\hline Subject & & Sand & Water & Ratio \\
\hline ID & (g) & $\begin{array}{r}\text { (CFU per } \\
\text { person) }\end{array}$ & $\begin{array}{r}\text { (CFU per } \\
\text { person) }\end{array}$ & $\begin{array}{l}\text { (sand/ } \\
\text { water) }\end{array}$ \\
\hline T1 & $<0.1$ & N.D. & $\mathrm{BDL}$ & $\mathrm{N} / \mathrm{A}$ \\
\hline $\mathrm{T} 2$ & 6.8 & 500 & $2.7 \times 10^{4}$ & $1.8 \%$ \\
\hline Т3 & 9.9 & $<6$ & $1.1 \times 10^{3}$ & $0.18 \%$ \\
\hline T4 & 12.7 & $<6$ & $1.3 \times 10^{3}$ & $0.19 \%$ \\
\hline T5 & 3.9 & $<6$ & $\mathrm{BDL}$ & N/A \\
\hline T6 & 24.4 & $<6$ & $6.3 \times 10^{4}$ & $0.01 \%$ \\
\hline T7 & 3.8 & $<6$ & $\mathrm{BDL}$ & N/A \\
\hline Т8 & 4.4 & $<6$ & $2.5 \times 10^{3}$ & $0.08 \%$ \\
\hline T9 & 6.5 & $<6$ & $\mathrm{BDL}$ & N/A \\
\hline T10 & 8.6 & 160 & $2.3 \times 10^{4}$ & $0.70 \%$ \\
\hline T11 & 3.7 & 200 & $4.5 \times 10^{5}$ & $0.04 \%$ \\
\hline $\mathrm{T} 12^{*}$ & 5.8 & $<6$ & $1.4 \times 10^{4}$ & $0.02 \%$ \\
\hline T13 & 10.4 & $<6$ & $2.3 \times 10^{3}$ & $0.10 \%$ \\
\hline T14 & 7.6 & 12 & $1.4 \times 10^{4}$ & $0.09 \%$ \\
\hline Average & 7.7 & 69 & $4.3 \times 10^{4}$ & $0.32 \%$ \\
\hline $\begin{array}{l}\text { Standard } \\
\text { Deviation }\end{array}$ & 5.8 & 145 & $1.2 \times 10^{5}$ & $0.09 \%$ \\
\hline
\end{tabular}

* Indicates the study participant colonized with MSSA.

$\mathrm{N} / \mathrm{A}=$ Not applicable. $\mathrm{BDL}=$ below detection limit. BDLs were transformed to $1 / 2$ of BDLs in order to estimate averages and standard deviations. The theoretical detection limit $\left(2.8 \times 10^{2} \mathrm{CFU} /\right.$ person) can be calculated from the volume of water poured on the toddler $(14 \mathrm{~L})$ and the largest membrane filtration volume $(50 \mathrm{ml})$.

Normalizing the results by the 10 adult participants in group II, MRSA shedding on a per person basis was 1.4 $\times 10^{4} \mathrm{CFU} /$ person for cycle $1,7.8 \times 10^{4} \mathrm{CFU} /$ person for cycle 3 , and $1.0 \times 10^{5} \mathrm{CFU} /$ person for cycle 4 as measured using BP selection; and $6.5 \times 10^{4} \mathrm{CFU} /$ person and $9.0 \times 10^{4} \mathrm{CFU} /$ person for cycles 3 and 4 , respectively, for samples evaluated using $\mathrm{CHR}$ selection. These values represent 15 to $20 \%$ of the total S. aureus observed in the pool water for Group II adults.

Only one of the toddlers, subject T12, was determined to have nasal colonization with MSSA; however, 10 of the 14 (71\%), including T12, had S. aureus isolated from their water samples. Thirteen of the subjects carried sufficient sand/sediment into the pool for evaluation; however, only 4 (31\%) of these were positive for MSSA, and this did not include subject T12 (Figure 2). All positive sand samples were associated with positive water samples, but only $40 \%$ ( 4 of 10 ) of the positive water samples were associated with sand; therefore, the sand did not account for the majority of MSSA shed from the toddlers not known to be colonized. In fact, the sand sample from the only toddler determined to be colonized was negative for MSSA. No nasal cultures from toddlers were positive for MRSA, and MRSA was not detected from any water or sediment samples from these participants. The lack of MRSA nasal colonization is consistent with the lack of MRSA in all of the sand and water samples from the toddler participants.

\section{Genetic characteristics}

SCCmec type, spa type and selected gene profiles (gyrA, $m e c \mathrm{~A}$ and $p v l$ ) are presented for all the MRSA isolated from colonized individuals $(\mathrm{n}=2)$, and water samples $(\mathrm{n}=15)$ and selected toxin gene profiles and spa type are presented for all MSSA from colonized individuals $(\mathrm{n}=3)$ and for a representative sample of corresponding

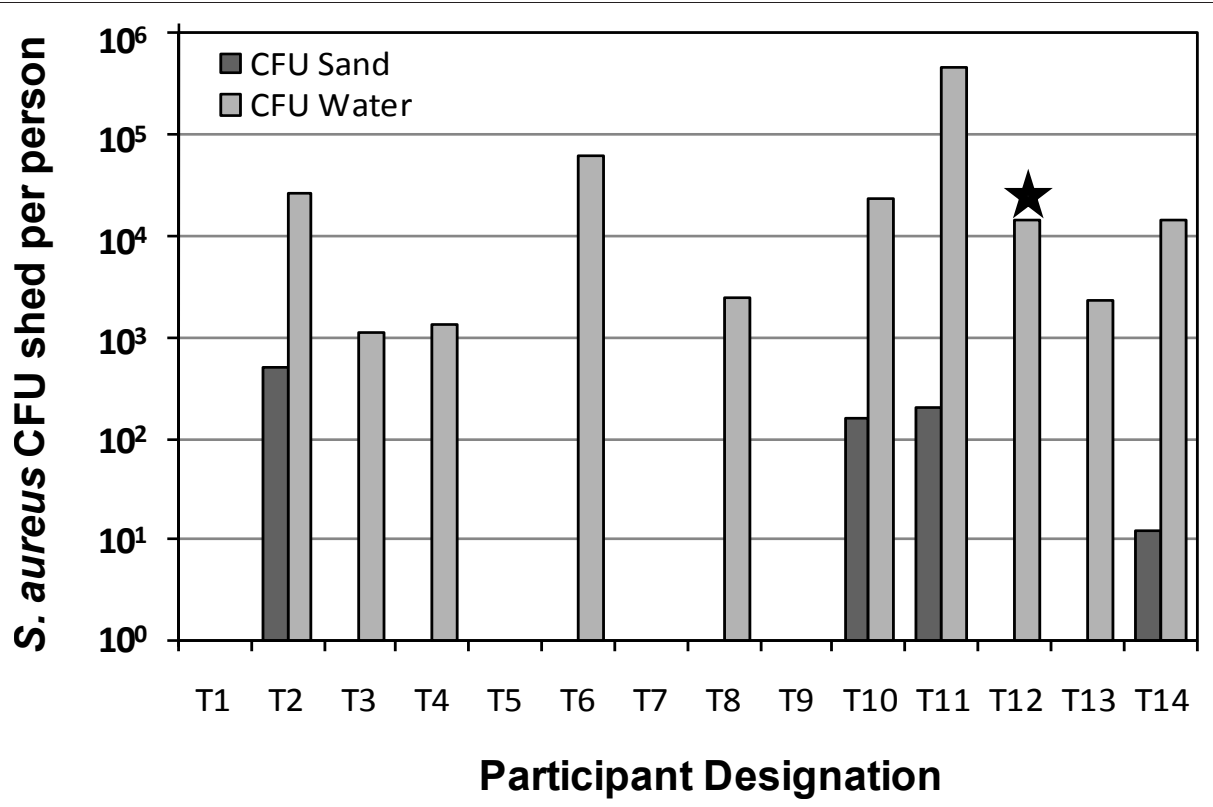

Figure 2 S. aureus CFU/person shed in small pool with individual toddlers. Star indicates participant with MSSA colonization. 
water isolates $(\mathrm{n}=17)$ (Table 3$)$. Among the MRSA, the 2 organisms isolated from the participants, and 12 of 15 of the MRSA from the water samples collected from the adult Group II study were identical by these analyses. The remaining 3 MRSA differed only in spa type. Given the nasal culture results and comparison of the genetic analysis, the MRSA likely came primarily from the subset of colonized individuals among the 10 adult participants in the pool. Therefore, the calculated amount of MRSA shedding per person could have been up to a factor of 5 higher (assuming that MRSA came from the two participants whose nasal cultures tested positive).

Similarly, MSSA with identical spa types were isolated from nares cultures and corresponding waters samples for the colonized toddler and the Group I adults (Table 3). Non-identical MSSA were also isolated from water samples of the colonized toddler (one of five) and from the Group I adult study (three of twelve), indicating the presence of organisms associated with individuals but not identified in the nares cultures. MSSA was also isolated from all water collections of the adult Group II study when no individuals were identified with MSSA colonization; this also indicated the presence of organisms associated with individuals but not identified in nares cultures, and likely represents colonization of participating individuals in areas of the body other than the nares.

\section{Discussion}

In these studies, we demonstrated that human bathers, both adults and toddlers in diapers, have the potential to release significant amounts of $S$. aureus (including MRSA) into the water column from direct shedding off their body and via sand transported on their skin. This suggests that recreational beaches may be potential exposure and transmission pathways for $S$. aureus (including MRSA). The authors hypothesize that the low background levels of MSSA in the off shore water was due to the residual effects from bather swimming activities from normal beach use given the potential persistence of these organisms in seawater [12]. These background levels, however, were very low in comparison to those levels observed during the small and large pool studies (which allowed for the quantification of the number of MSSA and MRSA released by the study participants).

The average quantities of $S$. aureus shed in this study were lower than those observed previously by Elmir et al. [17] using less stringent identification criteria. In addition to more stringent techniques, the difference in numbers may also be due to the differences in the degree to which the adults in the different studies were colonized by, and therefore shed, S. aureus. The shedding numbers reported above take into account the entire population, which included both those individuals who shed and those who did not shed bacteria. Therefore, individuals who participated in the large pool study who were not truly colonized, would not have contributed organisms to the pool water, yet were considered in the overall per person shedding calculations. However, when shedding was evaluated on an individual basis (as was done with the toddler study), the number of organisms shed could have been much higher per person if an adult bather in the group happened to have been colonized and was not detected by nares culture. This was the case in the adult Group II where no MSSA was isolated from participants directly, but MSSA was in the water during cycles 1 and 2 prior to sand exposure. This difference may also be due to variability of $S$. aureus shedding among different people depending upon their individual colonization status, body site colonized and quantity of organisms. Variable shedding by individuals was observed from the small pool study, where toddler shedding ranged from non-detectable levels up to values above $10^{5} \mathrm{CFU} /$ person.

Direct shedding of $S$. aureus from the body can include release from known colonized body areas such as the nares. This was seen when the large pool sample water (for Group II) was positive for MRSA only when MRSA was found in the anterior nares of participants who bathed in that water; and the majority of these organisms were shown to have the same genetic characteristics as the colonizing MRSA. Direct shedding was also observed when the single known nasally colonized toddler shed into the water sample in the small pool study. The results reported here confirm that $S$. aureus are shed by colonized adults and toddlers into the water column. This is supported by the results from both adults and toddlers in the separate pool studies. In the large pool studies, MSSA and MRSA were isolated when the participants were known only to be colonized with MRSA only (Group II); however, although only 1 toddler was shown to be colonized by nares sampling method, 10 toddlers shed MSSA. As a result of these findings, we hypothesize that both adults and toddlers are likely colonized with S. aureus, in particular MSSA, in other areas of the body, and that these locations contribute to bacterial shedding when exposed to water. This observation is consistent with clinical observations showing that about one third of MRSA-infected patients were not nasally colonized [25], with alternate colonization sites including skin [26] and throat [27].

Both the large pool study and the small pool study demonstrated that sand played a relatively small role in $S$. aureus shedding. In the small pool study during the single bathing cycle, sand accounted for less than $1 \%$ of shedding. Elmir et al. [18] also found that sand accounted for roughly $3.7 \%$ of the enterococci 
Table 3 Bather associated S. aureus: MSSA and MRSA, collected as shed organisms from toddlers and adults Toddler Shedding: Small individual pools

\begin{tabular}{|c|c|c|c|c|c|c|}
\hline Isolate & Source & gyrA & $\operatorname{mec} A$ & $p v l$ & SCCmec type & spa type \\
\hline BLP1347 & Toddler 12 nares & pos & neg & neg & NA & t874 \\
\hline BLP1275 & Toddler 12 pool & pos & neg & neg & NA & t874 \\
\hline BLP1276 & Toddler 12 pool & pos & neg & neg & NA & t874 \\
\hline BLP1277 & Toddler 12 pool & pos & neg & neg & NA & t411 \\
\hline BLP1278 & Toddler 12 pool & pos & neg & neg & NA & t874 \\
\hline BLP1279 & Toddler 12 pool & pos & neg & neg & NA & t874 \\
\hline \multicolumn{7}{|c|}{ Adult Shedding: Large shared pools Group 1} \\
\hline Isolate & Source & gyrA & mecA & pvl & SCCmec type & spa type \\
\hline BLP1207 & Group 1-Adult subject B-nares & pos & neg & neg & NA & t001 \\
\hline BLP1208 & Group 1-Adult subject A-nares & pos & neg & neg & NA & t001 \\
\hline BLP1295 & Group 1-cycle 1-pool & pos & neg & neg & NA & t001 \\
\hline BLP1296 & Group 1-cycle 1-pool & pos & neg & neg & NA & t001 \\
\hline BLP1297 & Group 1-cycle 1-pool & pos & neg & neg & NA & t001 \\
\hline BLP1309 & Group 1-cycle 2-pool & pos & neg & neg & NA & t001 \\
\hline BLP1310 & Group 1-cycle 2-pool & pos & neg & neg & NA & t001 \\
\hline BLP1311 & Group 1-cycle 2-pool & pos & neg & neg & NA & t001 \\
\hline BLP1317 & Group 1-cycle 3-pool & pos & neg & neg & NA & t001 \\
\hline BLP1318 & Group 1-cycle 3-pool & pos & neg & neg & NA & t001 \\
\hline BLP1319 & Group 1-cycle 3-pool & pos & neg & neg & NA & t001 \\
\hline BLP1361 & Group 1-cycle 4-pool & pos & neg & neg & NA & $\mathrm{t} 122$ \\
\hline BLP1362 & Group 1-cycle 4-pool & pos & neg & neg & NA & $\mathrm{t} 122$ \\
\hline BLP1363 & Group 1-cycle 4-pool & pos & neg & neg & NA & $\mathrm{t} 122$ \\
\hline \multicolumn{7}{|c|}{ Adult Shedding: Large shared pools Group 2} \\
\hline BLP1209 & Group 2-Adult subject C-nares & pos & pos & neg & IV & t007 \\
\hline BLP1210 & Group 2-Adult subject D-nares & pos & pos & neg & IV & t007 \\
\hline BLP1175 & Group 2-cycle 1-pool & pos & pos & neg & IV & t001 \\
\hline BLP1187 & Group 2-cycle 3-pool & pos & pos & neg & IV & t001 \\
\hline BLP1189 & Group 2-cycle 3-pool & pos & pos & neg & IV & t001 \\
\hline BLP1191 & Group 2-cycle 3-pool & pos & pos & neg & IV & t007 \\
\hline BLP1193 & Group 2-cycle 3-pool & pos & pos & neg & IV & t007 \\
\hline BLP1194 & Group 2-cycle 3-pool & pos & pos & neg & IV & t007 \\
\hline BLP1195 & Group 2-cycle 3-pool & pos & pos & neg & IV & t007 \\
\hline BLP1198 & Group 2-cycle 4-pool & pos & pos & neg & IV & t007 \\
\hline BLP1199 & Group 2-cycle 4-pool & pos & pos & neg & IV & t007 \\
\hline BLP1200 & Group 2-cycle 4-pool & pos & pos & neg & IV & t007 \\
\hline BLP1201 & Group 2-cycle 4-pool & pos & pos & neg & IV & t007 \\
\hline BLP1202 & Group 2-cycle 4-pool & pos & pos & neg & IV & t007 \\
\hline BLP1204 & Group 2-cycle 4-pool & pos & pos & neg & IV & t007 \\
\hline BLP1205 & Group 2-cycle 4-pool & pos & pos & neg & IV & t007 \\
\hline BLP1206 & Group 2-cycle 4-pool & pos & pos & neg & IV & t007 \\
\hline
\end{tabular}

Isolate designations presented with source of collection site and subject. PCR was used to determine presence of gyrA, S. aureus specific DNA gyrase A gene; mecA, methicillin resistance gene; $p v l$ genes encoding Panton-Valentine leukocidin and Staphylococcal cassette chromosome mec type (SCCmec) Staphylococca protein A type, spa type are shown. 
contribution in the first bathing cycle for the small pool study. For the large pool study, an increase in $S$. aureus shedding was observed when participants were exposed to sand between the second and third bathing cycles, but the impacts were less pronounced for S. aureus as compared to enterococci shedding as observed in prior studies [18]. Increased numbers of $S$. aureus shed in the third cycle could be associated with sand exposures; however, the ultimate source of the $S$. aureus in the sand is unknown, and may be associated with naturally existing $S$. aureus and/or from direct shedding from humans to the sand.

Because of the differences in the designs of the large pool study (adults) and the small pool study (toddlers), direct comparison of the amount of shedding between toddlers and adults in this study is limited. Nevertheless, we compared the numbers of $S$. aureus shed by adult and toddlers, keeping these limitations in mind. The average of $S$. aureus shed by adults during the four cycles in the large pool ( $\mathrm{n}=8$ composites of 10 people) was $6.3 \times 10^{5}$ $\mathrm{CFU} /$ person, and by toddlers $(\mathrm{n}=14)$ was $4.3 \times 10^{4} \mathrm{CFU} /$ person in the small pool. In this comparison, adults shed 13 times more $S$. aureus than toddlers on average (75 times on median). It should be noted that the estimated adult body surface areas based on height and weight [28] were approximately 3.5 times larger than the toddlers in this study. After normalizing shedding numbers by the body surface factor of 3.5, the numbers of $S$. aureus shed by adults were 4 times more than toddlers on average (21 times on median). Therefore, in this investigation, toddlers in diapers shed fewer organisms than the adults; however, additional studies need to be done under the same conditions to confirm these findings.

\section{Conclusions}

The results of this study showed that both MSSA and MRSA were shed by human populations into marine waters. The amount of shedding varied, was likely dependent upon the level of colonization of the host, and colonization was not limited to the anterior nares. In this study, the shedding of MRSA was directly dependent upon its colonization of the human host. MRSA shedding was observed intermittently, only among Group II adults and water, with the apparent lower number of humans colonized by MRSA relative to MSSA. No MRSA was observed in the sand samples as the pediatric populations evaluated in this study were apparently not colonized with MRSA. However, it is highly likely that similar studies with additional pediatric participants would result in the isolation of MRSA [29]. Future studies should focus on the collection of additional samples from human participants as the current study was limited by the restricted numbers of carriers identified. These future studies should collect samples from the skin and from other areas where $S$. aureus resides, in addition to samples from the anterior nares.

Once $S$. aureus is released from bathers, its potential for transmission is highly dependent on its persistence in the environment. Gregg and LaCroix [30] inoculated saltwater pool water with MRSA, and found very low levels after 1 hour exposure. They concluded that swimming pool water would not likely put children at risk for acquiring MRSA. However, we argue here that more research is needed to evaluate the risk of illness associated with water exposures and the potential for transmission through sand, including the residency time of these human pathogens in both recreational marine waters and beach sand [12]. Future research should be conducted with $S$. aureus species from actively colonized individuals, as the current study found large amounts released from individuals, and the actively growing clinical strains may survive differently in comparison to laboratory grown strains used for inoculation experiments. Experimentation should closely control environmental factors as some studies have documented growth of $S$. aureus under optimal environmental conditions [31].

Overall, the results from this study confirmed that both adults and toddlers can be sources of potentially pathogenic MSSA and MRSA in recreational marine waters, and support the potential for exposure and transmission of these organisms through the use of recreational beaches. More research is needed to evaluate the persistence of strains released by humans into the water column and the possibility of disease transmission at recreational beach sites.

\section{Acknowledgements}

This study was funded in part from the following sources: the National Institute of Environmental Health Sciences (NIEHS) Oceans and Human Health Center at the University of Miami Rosenstiel School (NSF OCE0432368/0911373; NIEHS 1 P50 ES12736) and NSF REU in Oceans and Human Health, and the National Science Foundation (NSF SGER 0743987) in Oceans and Human Health, the University of Miami IRDI program, the National Center for Environmental Health (NCEH), Centers for Disease Control and Prevention (CDC); Florida Dept of Health (FL DOH) through monies from the Florida Dept of Environmental Protection (FL DEP) and the Environmental Protection Agency (EPA) Internship Program. The research team gratefully acknowledges all organizations and their staff who collaborated, provided support, and/or participated in all various aspects of this research effort including: University of Miami, Florida International University, University of Florida, Miami Dade County Public Works, Miami Dade County Health Department Environmental Health, Florida Department of Health Bureau of Laboratory Services Miami Branch, US Department of Commerce National Oceanic and Atmospheric Administration, and U.S. Department of Health Human Services (DHHS). Finally, the researchers would like to thank Ms Kathy Vergara (Director), the Staff and the families of the Debbie School of the University of Miami for their support of and participation in this study.

\section{Author details}

${ }^{1}$ Department of Pediatrics and Department of Microbiology and Immunology, University of Miami, Miami, Florida 33130 USA. ${ }^{2}$ NSF-NIEHS Oceans and Human Health Center, University of Miami, Rosenstiel School for 
Marine and Atmospheric Sciences, 4600 Rickenbacker Causeway, EG 211 Key Biscayne, FL 33149 USA. ${ }^{3}$ NOAA Atlantic Oceanographic and Meteorological Laboratory, Miami, FL 33149 USA. ${ }^{\text {P }}$ ublic Health and Health Education Programs, Northern Illinois University, DeKalb, IL USA. ${ }^{5}$ Miami-Dade County Health Department, 1725 NW 167 Street Miami, Florida 33056, USA.

${ }^{6}$ University of Miami, Department of Epidemiology and Public Health, 1120 NW 14th Street, Room 1049, Miller School of Medicine, Miami, FL 33136 USA. 'University of Miami, Department of Civil, Architectural, and Environmental Engineering, P.O. Box 248294, Coral Gables, Florida, 331240630, USA.

\section{Authors' contributions}

LRWP with ACG, CDS, MLG, and TS performed all the laboratory analyses and with SME, JK, GM, KW, HMSG, and LEF performed all the field studies. LRWP, JK, LEF, TS, and HMSG performed all the statistical analyses. All authors contributed to and edited the manuscript.

Received: 7 September 2010 Accepted: 6 January 2011

Published: 6 January 2011

\section{References}

1. Kluytmans J, van Belkum A, Verbrugh $\mathrm{H}$ : Nasal carriage of Staphylococcus aureus: epidemiology, underlying mechanisms, and associated risks. Clinical Microbiology Reviews 1997, 10:505-520.

2. Cole AM, Tahk S, Oren A, Yoshioka D, Kim YH, Park A, Ganz T: Determinants of Staphylococcus aureus nasal carriage. Clinical and diagnostic laboratory immunology 2001, 8:1064-1069.

3. von Eiff C, Becker K, Machka K, Stammer H, Peters G: Nasal carriage as a source of Staphylococcus aureus bacteremia. Study Group. The New England Journal of Medicine 2001, 344:11-16.

4. Diep BA, Carleton HA, Chang RF, Sensabaugh GF, Perdreau-Remington F: Roles of 34 virulence genes in the evolution of hospital- and community-associated strains of methicillin-resistant Staphylococcus aureus. The Journal of infectious diseases 2006, 193:1495-1503.

5. Klevens RM, Morrison MA, Nadle J, Petit S, Gershman K, Ray S, Harrison LH, Lynfield R, Dumyati G, Townes JM, Craig AS, Zell ER, Fosheim GE, McDougal LK, Carey RB, Fridkin SK: Invasive methicillin-resistant Staphylococcus aureus infections in the United States. JAMA 2007, 298:1763-1771.

6. Herold BC, Immergluck LC, Maranan MC, Lauderdale DS, Gaskin RE, BoyleVavra S, Leitch CD, Daum RS: Community-acquired methicillin-resistant Staphylococcus aureus in children with no identified predisposing risk. JAMA 1998, 279:593-598.

7. Eady EA, Cove JH: Staphylococcal resistance revisited: communityacquired methicillin resistant Staphylococcus aureus-an emerging problem for the management of skin and soft tissue infections. Curr Opin Infect Dis 2003, 16:103-124.

8. Moran GJ, Krishnadasan A, Gorwitz RJ, Fosheim GE, McDougal LK, Carey RB, Talan DA: Methicillin-resistant S. aureus infections among patients in the emergency department. The New England Journal of Medicine 2006, 355:666-674.

9. Charoenca N, Fujioka R: Assessment of staphylococcus bacteria in Hawaii marine recreational waters. Water Sci Technol 1993, 27:283-289.

10. Charoenca N, Fujioka RS: Association of staphylococcal skin infections and swimming. Water Science and Technology 1995, 31:11-17.

11. Gabutti G, De Donno A, Bagordo F, Montagna MT: Comparative Survival of Faecal and Human Contaminants and Use of Staphylococcus aureus as an Effective Indicator of Human Pollution. Marine Pollution Bulletin 2000, 40:697-700.

12. Fujioka RS, Unutoa TM: Comparative stability and growth requirements of S. aureus and faecal indicator bacteria in seawater. Water Sci Technol 2006, 54:169-175.

13. Tice AD, Pombo D, Hui J, Kurano M, Bankowski MJ, Seifried SE: Quantitation of Staphylococcus aureus in seawater using CHROMagar SA. Hawaii Med J 2010, 69(1):8-12.

14. Goodwin KD, Pobuda M: Performance of CHROMagar Staph aureus and CHROMagar MRSA for detection of Staphylococcus aureus in seawater and beach sand-comparison of culture, agglutination, and molecular analyses. Water Research 2009, 43:4802-4811.

15. Soge OO, Meschke JS, No DB, Roberts MC: Characterization of methicillinresistant Staphylococcus aureus and methicillin-resistant coagulase- negative Staphylococcus spp. isolated from US West Coast public marine beaches. J Antimicrob Chemother 2009, 64:1148-1155.

16. Abdelzaher AM, Wright ME, Ortega C, Solo-Gabriele HM, Miller G, Elmir S, Newman X, Shih P, Bonilla JA, Bonilla TD, Palmer CJ, Scott T, Lukasik J, Harwood VJ, McQuaig S, Sinigalliano C, Gidley M, Plano LR, Zhu X, Wang JD, Fleming LE: Presence of pathogens and indicator microbes at a non-point source subtropical recreational marine beach. Applied and environmental microbiology 2010, 76:724-732.

17. Elmir SM, Wright ME, Abdelzaher A, Solo-Gabriele HM, Fleming LE, Miller G, Rybolowik M, Peter Shih MT, Pillai SP, Cooper JA, Quaye EA: Quantitative evaluation of bacteria released by bathers in a marine water. Water Research 2007, 41:3-10

18. Elmir SM, Shibata T, Solo-Gabriele HM, Sinigalliano CD, Gidley ML, Miller G, Plano LR, Kish J, Withum K, Fleming LE: Quantitative evaluation of enterococci and Bacteroidales released by adults and toddlers in marine water. Water Research 2009, 43:4610-4616.

19. United States Environmental Protection Agency (EPA): EPA Method 1600: membrane filter test method for enterococci in water 2002, EPA-821-R-02-022.

20. Böcher S, Smyth R, Kahlmeter G, Kerremans J, Vos MC, Skov R: Evaluation of Four Selective Agars and Two Enrichment Broths in Screening for Methicillin-Resistant Staphylococcus aureus. Journal of Clinical Microbiology 2008, 46(9):3136-3138.

21. Mertz PM, Cardenas TC, Snyder RV, Kinney MA, Davis SC, Plano LR Staphylococcus aureus virulence factors associated with infected skin lesions: influence on the local immune response. Archives of Dermatology 2007, 143:1259-1263.

22. Oliveira DC, Crisostomo I, Santos-Sanches I, Major P, Alves CR, Aires-deSousa M, Thege MK, de Lencastre H: Comparison of DNA sequencing of the protein A gene polymorphic region with other molecular typing techniques for typing two epidemiologically diverse collections of methicillin-resistant Staphylococcus aureus. Journal of Clinical Microbiology 2001, 39:574-580.

23. Shopsin B, Gomez M, Montgomery SO, Smith DH, Waddington M, Dodge DE, Bost DA, Riehman M, Naidich S, Kreiswirth BN: Evaluation of protein A gene polymorphic region DNA sequencing for typing of Staphylococcus aureus strains. Journal of Clinical Microbiology 1999, 37:3556-3563.

24. Shibata T, Solo-Gabriele HM, Fleming LE, Elmir S: Monitoring marine recreational water quality using multiple microbial indicators in an urban tropical environment. Water Research 2004, 38:3119-3131.

25. Robicsek A, Suseno M, Beaumont JL, Thomson RB Jr, Peterson LR: Prediction of methicillin-resistant Staphylococcus aureus involvement in disease sites by concomitant nasal sampling. J Clin Microbiol 2008, 46(2):588-592.

26. Chung HJ, Jeon HS, Sung H, Kim MN, Hong SJ: Epidemiological characteristics of methicillin-resistant Staphylococcus aureus isolates from children with eczematous atopic dermatitis lesions. J Clin Microbiol 2008, 46(3):991-995.

27. Widmer AF, Mertz D, Frei R: Necessity of screening of both the nose and the throat to detect methicillin-resistant Staphylococcus aureus colonization in patients upon admission to an intensive care unit. J Clin Microbiol 2008, 46(2):835.

28. United States Environmental Protection Agency: Exposure Factor Handbook U.S. EPA. National Center for Environmental Assessment Washington, D.C; 1997.

29. Simor AE, Gilbert NL, Gravel D, Mulvey MR, Bryce E, Loeb M, Matlow A, McGeer A, Louie L, Campbell J: Methicillin-resistant Staphylococcus aureus colonization or infection in Canada: National Surveillance and Changing Epidemiology, 1995-2007. Infect Control Hosp Epidemiol 2010, 31:348-356.

30. Gregg M, Lacroix R: Survival of community-associated methicillin-resistant Staphylococcus aureus in 3 different swimming pool environments (chlorinated, saltwater, and biguanide nonchlorinated). Clin Pediatr (Phila) 2010, 49(7):635-7.

31. Valero A, Pérez-Rodríguez F, Carrasco E, Fuentes-Alventosa JM, GarcíaGimeno RM, Zurera G: Modelling the growth boundaries of Staphylococcus aureus: Effect of temperature, $\mathrm{pH}$ and water activity. Int $J$ Food Microbiol 2009, 133(1-2):186-194.

doi:10.1186/1471-2180-11-5

Cite this article as: Plano et al: Shedding of Staphylococcus aureus and methicillin-resistant Staphylococcus aureus from adult and pediatric bathers in marine waters. BMC Microbiology 2011 11:5. 